\title{
PRODUÇÃO E CARACTERÍSTICAS QUALITATIVAS DOS FRUTOS DE HÍBRIDOS DE MARACUJAZEIRO-AMARELO ${ }^{1}$
}

\author{
PAULO VICENTE CONTADOR ZACCHEO ${ }^{2}$, RICARDO SFEIR DE AGUIAR ${ }^{3}$, \\ NEUSA MARIA COLAUTO STENZEL ${ }^{4}$, TUMORU SERA ${ }^{5}$, \\ CARMEN SILVIA VIEIRA JANEIRO NEVES ${ }^{6}$
}

RESUMO-A pequena disponibilidade de genótipos melhorados de maracujazeiro-amarelo contribui para a baixa produtividade da cultura no Brasil. Materiais genéticos devem ser desenvolvidos visando ao incremento da produtividade e da qualidade dos frutos. O objetivo deste trabalho foi avaliar a produção e as características qualitativas dos frutos de 36 híbridos de maracujazeiro-amarelo cultivados no norte do Paraná. As avaliações dos híbridos foram realizadas durante dois ciclos de produção da cultura, de abril a julho de 2007 e de janeiro a julho de 2008, com coletas semanais dos frutos maduros. A produção foi determinada a partir da produção total e do número de frutos por planta. Para as características qualitativas, foram avaliadas: massa, comprimento, diâmetro e relação entre comprimento e diâmetro dos frutos, além de espessura da casca, rendimento de polpa, teor de sólidos solúveis totais (SST), acidez total titulável (ATT) e relação SST/ATT. A produção, em quilos de fruta por planta, situou-se entre 20 e 40 no primeiro ciclo e 54 a 96 no segundo, o que representa uma produtividade de 30 a 53 ton ha ${ }^{-1}$ para o conjunto das duas safras. Os híbridos '40' e '43'apresentam, respectivamente, maior produção e maior número de frutos no primeiro ciclo de produção Os frutos do híbrido ' 36 ' destacaram-se no atendimento das caracteristicas desejáveis de qualidade para o consumo in natura, enquanto os híbridos ' 43 ' e ' 60 ' apresentaram caracteristicas importantes para a indústria de sucos.

Termos para indexação: Passiflora edulis, qualidade do fruto, heterose.

\section{YIELD AND FRUIT QUALITATIVE CHARACTERISTICS OF PASSION FRUIT HYBRIDS}

\begin{abstract}
The small number of improved genotypes of passion fruit contributes to the low productivity of this crop in Brazil. New genetic materials should be developed to increase yield and fruit quality. The aim of this study was to evaluate the yield and the qualitative characteristics of 36 passion fruit hybrids grown in northern Paraná. The evaluations were carried out during two production cycles, from April to June 2007 and from January to July 2008, by weekly harvests of ripe fruits. The yield was determined by total yield and number of fruits per plant. For the fruit quality the following characteristics were evaluated: weight, length, diameter and relationship between length and diameter of the fruits, skin thickness, pulp yield, total soluble solids (TSS), titratable acidity (TA) and ratio TSS/TA. Hybrid '36' presented fruits with desirable characteristics for the fresh market, while the hybrids ' 43 ' e ' 60 ' showed important features for the juice industry. Fruit yield, in kilograms of fruit per plant, stood between 20 and 40 in the first cycle and 54 to 96 in the second, which represents a yield of 30 to 53 ton ha- ${ }^{-1}$ for the two crop seasons together.
\end{abstract}

Index terms: Passiflora edulis, fruit quality, heterosis.

\footnotetext{
${ }^{1}$ (Trabalho 093-12). Recebido em: 08-02-2012. Aceito para publicação em: 28-08-2012.

${ }^{2}$ Eng. Agr. MSc. Doutorando do Programa de Pós graduação em Agronomia, Universidade Estadual de Londrina, Londrina-PR. E-mail: pvczaccheo@yahoo.com.br

${ }^{3}$ Eng. Agr. MSc. Doutorando do Programa de Pós graduação em Agronomia, Universidade Estadual de Londrina, Londrina-PR. E-mail: rsaguiar@hotmail.com

${ }^{4} \mathrm{Eng}^{\mathrm{a}}$. Agr $r^{\mathrm{a}}$. Dr ${ }^{\mathrm{a}}$. Pesquisadora do Instituto Agronômico do Paraná, Londrina-PR. E-mail: nstenzel@iapar.br

${ }^{5}$ Eng. Agr. Dr. Pesquisador do Instituto Agronômico do Paraná, Londrina-PR. E-mail: tsera@iapar.br

${ }^{6} \mathrm{Eng}^{\mathrm{a}}$. Agr ${ }^{\mathrm{a}}$. Dr ${ }^{\mathrm{a}}$. Professora da Universidade Estadual de Londrina, Londrina-PR. E-mail: csvjneve@uel.br
} 


\section{INTRODUÇÃO}

O maracujazeiro é originário da América Tropical e compreende mais de 150 espécies de Passifloráceas que podem ser utilizadas para consumo humano (LIMA; CUNHA, 2004). No Brasil, maior produtor mundial, a espécie mais representativa é o maracujazeiro-amarelo (Passiflora edulis Sims.), com aproximadamente $95 \%$ da área plantada (POLL et al., 2011). As principais demandas pelo fruto são provenientes, principalmente, da indústria de suco processado e do mercado de frutas frescas (AGUIAR; SANTOS, 2001).

As características desejáveis nos frutos dependem das necessidades de cada segmento. Para a indústria de sucos, as variedades devem apresentar bom rendimento de polpa, alta acidez, coloração do suco amarelo-dourada e alto teor de sólidos solúveis totais. Para o mercado de frutas frescas, priorizam-se frutos grandes e ovais, o que garante boa classificação comercial e boa aparência, visto serem estas característica preferidas pelo mercado consumidor (BRUCKNER et al., 2002).

Além das características qualitativas dos frutos, a produtividade é fundamental para atender às necessidades do mercado e à remuneração dos produtores. Entretanto, a produtividade média da cultura do maracujazeiro no Brasil é baixa, apresentando valores próximos a $14 \mathrm{t}$ por ha ano ${ }^{-1}$ (IBGE, 2011), o que se deve, entre outros fatores, à baixa disponibilidade e utilização de cultivares melhoradas (GONÇALVES et al., 2007). Atualmente, para a espécie Passiflora edulis Sims., existem apenas quatro cultivares registradas junto ao Registro Nacional de Cultivares do Ministério da Agricultura, Pecuária e Abastecimento, denominadas 'Amarelo', 'IAC Paulista', 'Redondo Amarelo' e 'Sol' (BRASIL, 2012).

Esses fatores, somados à grande variabilidade existente nos pomares comerciais, resulta na necessidade de obtenção de novas cultivares adaptadas às regiões produtoras. Assim, o objetivo deste trabalho foi avaliar a produção e as características qualitativas dos frutos de trinta e seis híbridos de maracujazeiro-amarelo cultivados no norte do Paraná.

\section{MATERIAL E MÉTODOS}

O experimento foi conduzido no município de Londrina-PR, na área experimental do Instituto Agronômico do Paraná (IAPAR), situado a $23^{\circ} 22^{\text {' }}$ $\mathrm{S}$ e $51^{\circ} 10^{\prime} \mathrm{W}$, com altitude de $585 \mathrm{~m}$, nas safras de 2007 e 2008. O clima, segundo a classificação de Wilhelm Köeppen, é subtropical úmido (Cfa), com precipitação média anual de $1.500 \mathrm{~mm}$, temperatura média anual de $22,8^{\circ} \mathrm{C}$, evapotranspiração anual de $1.350 \mathrm{~mm}$, umidade relativa anual média de $75 \%$ e insolação média de 7,14 $\mathrm{h} \mathrm{dia}^{-1}$ (CAVIGLIONE et al., 2000). O solo é classificado, de acordo com Bhering (2007), como Latossolo Vermelho distroférrico, de textura argilosa.

O delineamento experimental utilizado foi o de blocos ao acaso, com quatro repetições, e uma planta por parcela. Os tratamentos constituíram-se de 36 híbridos de maracujazeiro-amarelo provenientes de cruzamentos realizados em 2005 e 2006, com parentais selecionados de acessos do IAPAR, os quais apresentavam boas características agronômicas e cujas mudas foram produzidas a partir de sementes obtidas em frutos de plantios comerciais dos Estados do Paraná e de São Paulo. Cada híbrido recebeu um número de identificação, variando de 29 a 64.

Para a produção das mudas, foram utilizadas sementes provenientes de frutos sadios e maduros. As mudas foram formadas em sacos plásticos individuais, com volume de $500 \mathrm{~cm}^{3}$, e mantidas em viveiro telado até o transplante. Em setembro de 2006, com cerca de 120 dias após a semeadura, as mudas foram transplantadas para o campo, com espaçamento de 6,0 $\mathrm{m}$ na linha e 4,0 $\mathrm{m}$ na entrelinha (417 plantas $\left.\mathrm{ha}^{-1}\right)$. Esse espaçamento foi utilizado com o objetivo de facilitar a avaliação das parcelas. O sistema de condução e sustentação das plantas foi em espaldeira vertical, com os mourões a uma distância de $6,0 \mathrm{~m}$ e um fio de arame liso a 1,8 $\mathrm{m}$ de altura em relação ao solo. As plantas foram conduzidas em haste única até o fio de arame, e os ramos secundários, orientados para única direção. As plantas não foram polinizadas artificialmente, a área não foi irrigada e o controle de plantas daninhas foi realizado por meio de roçada das entrelinhas e capina manual nas linhas.

As avaliações dos híbridos foram realizadas durante os ciclos de produção da cultura, que corresponderam aos períodos de abril a julho de 2007 $\left(1^{\circ}\right.$ ciclo) e de janeiro a julho de 2008 ( $2^{\circ}$ ciclo), por meio da coleta semanal dos frutos maduros. A produção foi determinada a partir da produção total $(\mathrm{kg}$ planta $\left.^{-1}\right)$ e do número de frutos por planta. Para as características qualitativas, foram avaliados: massa média $(\mathrm{g})$, comprimento médio $(\mathrm{mm})$, diâmetro médio $(\mathrm{mm})$ e relação entre comprimento e diâmetro dos frutos, além de espessura média da casca $(\mathrm{mm})$, rendimento de polpa (\%), teor de sólidos solúveis totais - SST ( ${ }^{\circ}$ Brix), acidez total titulável - ATT $(\%)$ e relação SST/ATT.

As avaliações correspondentes às características qualitativas dos frutos foram realizadas em dez frutos por parcela, colhidos aleatoriamente durante cada ciclo de produção. Os dados de comprimento, 
diâmetro e espessura foram obtidos com paquímetro digital, com precisão de $0,1 \mathrm{~mm}$. Para determinar a espessura da casca, foram tomadas três medições em pontos equidistantes da casca de frutos cortados transversalmente, obtendo-se uma média por fruto.

Para a determinação do rendimento de polpa, foi calculada a porcentagem da massa da polpa, subtraindo-se a massa da casca e das sementes em relação à massa total do fruto. O teor de SST foi obtido através de refratômetro digital, com compensação automática de temperatura, e a ATT foi determinada através de titulação com $\mathrm{NaOH}$ a $0,1 \mathrm{~mol} \mathrm{~L}^{-1}$, expressa em percentagem de ácido cítrico, segundo técnicas preconizadas pela AOAC (1990).

Os dados foram submetidos à análise de variância complementada pelo teste de Tukey, a 5\% de significância. Os dados de produção e de massa média dos frutos foram comparados separadamente para cada ciclo de produção e acumulado dos dois ciclos. As demais variáveis foram analisadas e comparadas a partir das médias dos dois ciclos. Foi realizada transformação angular dos dados de rendimento de polpa.

\section{RESULTADOS E DISCUSSÃO}

Houve diferença significativa entre os híbridos para a produção do $1^{\circ}$ ciclo (Tabela 1 ), na qual o híbrido ' 40 ' se destacou com produção superior aos híbridos '31', '49', '51' e '59'. No $2^{\circ}$ ciclo e na produção acumulada, não foram verificadas diferenças significativas. Considerando a densidade populacional do maracujazeiro-amarelo no presente trabalho, estima-se que os híbridos produziram, no conjunto dos dois ciclos, de 30 a $53 \mathrm{t} \mathrm{ha}^{-1}$, valores acima da produção média brasileira, que é de $28 \mathrm{tha}^{-1}$ para dois ciclos (IBGE, 2011). Os valores referentes à produção obtidos neste trabalho devem-se, em grande parte, às boas características complementares dos componentes fisiológicos da produtividade dos parentais. Expressivas variações na produção de diferentes genótipos de maracujazeiro também foram observadas por Maia et al. (2009) e Melo et al. (2001) em experimentos realizados no Distrito Federal.

Na comparação do número de frutos produzidos, também houve diferença significativa no $1^{\circ}$ ciclo de produção (Tabela 1), sendo que o híbrido '43' produziu 228 frutos por planta, superando os híbridos ' 31 ', '49' e '51', que tiveram produção de 102 a 111 frutos por planta. Maia et al. (2009), avaliando o desempenho agronômico de 14 genótipos de maracujazeiro-amarelo no Distrito Federal, em clima tropical com estação seca de inverno, observaram valores menores e variações ainda mais expressivas para número de frutos. Naquelas condições, no $1^{\circ}$ ciclo de produção, o número de frutos produzidos variou de 30 a 128 frutos por planta. Por outro lado, além de verificar o número de frutos produzidos, é importante o registro da massa por fruto (Tabela 1), fator que afeta o valor da produção, por estar relacionado à qualidade, e que pode ser influenciado pelo volume da produção.

Pode-se inferir que, possivelmente, a diferença significativa entre os híbridos para os dados de produção observada no $1^{\circ}$ ciclo e a ausência de diferença no $2^{\circ}$ se deve à melhor adaptação ao ambiente e a uma rápida superação do estresse provocado pelo transplante daqueles híbridos que tiveram melhor desempenho em relação aos demais, pois, mesmo considerando o volume de $500 \mathrm{~cm}^{3}$ dos recipientes, já havia raízes mais longas, que tiveram de ser podadas para o plantio. Esse fator é importante para os produtores, que desejam o retorno mais rápido possível dos recursos investidos na implantação do pomar. No $2^{\circ}$ ciclo, com aproximadamente dois anos de idade, todos os materiais estavam adaptados ao clima, minimizando assim as possíveis diferenças observadas na safra anterior.

Para a massa dos frutos, foram evidenciadas diferenças significativas nos dois ciclos e na média dos dois ciclos de produção (Tabela 1). Nas três comparações, o híbrido ' 63 ' destacou-se, com massa média de frutos de 199 a $205 \mathrm{~g}$, sendo superior aos híbridos '29' e '61', que tiveram massa de 144 e $138 \mathrm{~g}$ no $1^{\circ}$ ciclo, aos híbridos ' 43 ' e ' $57^{\prime}$ ', que apresentaram massa de 139 e $133 \mathrm{~g}$ no $2^{\circ}$ ciclo e aos híbridos ' 35 ' e ' 61 ', que tiveram massa de 130 e $141 \mathrm{~g}$ na média dos dois ciclos. Isso faz com que o híbrido '63' seja uma interessante opção para o mercado de fruta fresca, que remunera melhor os frutos de maior tamanho (BRUCKNER et al., 2002). Meletti et al. (2000), avaliando caracteres produtivos de 8 híbridos de maracujazeiro-amarelo no interior de São Paulo, observaram valores de massa de fruto semelhantes aos do presente trabalho, os quais variaram de 155 a $237 \mathrm{~g}$.

Com relação aos caracteres físico-químicos dos frutos, não houve diferenças significativas para comprimento, diâmetro e teor de SST. Porém, para a relação entre comprimento e diâmetro, espessura da casca, rendimento de polpa, ATT e relação entre SST e ATT, foram observadas diferenças entre os híbridos (Tabela 2). Para a relação entre comprimento e diâmetro, o híbrido ' 36 ' apresentou-se com o formato mais oval (maior relação entre comprimento e diâmetro) em comparação aos híbridos ' 32 ', ' 45 ', ' 50 ' e ' 56 '. Essa característica é interessante, pois a preferência do mercado consumidor de fruta fresca é por frutos de formato ovalado (MELETTI et al., 
2000). A maior espessura de casca foi observada no híbrido '32', e a menor, no híbrido '54', com 7,6 e $3,3 \mathrm{~mm}$,respectivamente. Cascas muito espessas resultam em menor rendimento de polpa, porém, para o transporte, essa característica torna-se uma vantagem, por aumentar a resistência física do fruto (MELETTI et al., 2000). Os híbridos '43’e '60' apresentaram maior rendimento de polpa, com valores de 35,5 e 34,9\%, em comparação ao híbrido ' 42 ', o que é interessante do ponto de vista do rendimento das indústrias de suco (BRUCKNER et al., 2002). Para a ATT, também importante característica do ponto de vista industrial, o híbrido ' 43 ' superou os híbridos '32', '35', '40', '41', '42', '51', '53’e ' 58 ', e, consequentemente, apresentou menor valor de relação SST/ATT do que os híbridos '32', '38', '41', '42', '46'e '53'.

Para o mercado in natura, o tamanho dos frutos é uma característica muito apreciada pelos consumidores. Todos os híbridos do presente trabalho produziram frutos com diâmetro maior que $55 \mathrm{~mm}$ (Tabela 2), considerado o mínimo adequado para se enquadrar na classificação comercial (MAIA et al., 2009).

Os frutos destinados à indústria de suco devem apresentar, preferencialmente, alto rendimento de polpa, alto teor de SST e alta ATT (BRUCKNER et al., 2002; NASCIMENTO et al., 1999). O alto teor de SST possibilita o uso de menor quantidade de frutos para concentração do suco, e a elevada acidez garante maior flexibilidade na adição de açúcares (SOUZA; SANDI, 2001).

Os híbridos '43' e '60' alcançaram valores para o rendimento de polpa variando de 34,9 a $35,5 \%$ (Tabela 2), o que é interessante, pois segundo Araújo et al. (1974), os frutos de maracujazeiro-amarelo destinados à industria devem ter rendimento de polpa de, no mínimo, 30\%. Menores rendimentos de polpa foram observados por Abreu et al. (2009) nas condições do Distrito Federal. Porém, Negreiros et al. (2008), Pio et al. (2003), Godoy et al. (2007) e Nascimento et al. (2003), ao avaliarem diferentes genótipos, observaram valores máximos de 45,8; 58,$5 ; 52,4$ e $57,7 \%$, respectivamente.

A maioria dos híbridos, neste trabalho, apresentou teores de SST dos frutos dentro da faixa esperada para frutos de maracujazeiro-amarelo (Tabela 2), que varia de 12,5 a $14,9^{\circ} \mathrm{Brix}$ (DURIGAN et al., 2004) e, consequentemente, atendem ao padrão mínimo de $13{ }^{\circ}$ Brix exigido pelas agroindústrias de sucos (MELETTI et al., 2000). Estes valores estão próximos daqueles observados por Meletti et al. (2000), avaliando oito híbridos no interior de São Paulo, e por Pio et al. (2003), que estudaram sete seleções em Lavras - MG, e que constataram teores de SST de 14 a $16^{\circ}$ Brix. Contudo, embora satisfatórios, os teores de SST determinados são menores do que os observados em outros genótipos de maracujazeiro-amarelo. Negreiros et al. (2008) observaram valores de até $17^{\circ}$ Brix ao avaliarem diferentes genótipos nas condições do Estado do Acre. Junqueira et al. (2010), nas condições do Cerrado brasileiro, e Nascimento et al. (2003) na Amazônia Oriental, também determinaram que os acessos avaliados tinham, em média, teor de SST de 15,9 e $16,2^{\circ}$ Brix, respectivamente. Porém, essas diferenças podem ser creditadas à maior temperatura média do ar durante a formação e o amadurecimento dos frutos, pois essa característica do ambiente está diretamente relacionada à produção de açúcares pela planta (VERAS et al., 2000).

Os valores de ATT observados nos frutos estão acima de 2,5\% (Tabela 2), que é o padrão mínimo para suco de maracujá estabelecido pelo Ministério da Agricultura no Brasil (BRASIL, 2003). Nascimento et al. (2003) obtiveram valor médio para ATT de $3,4 \%$, e valores máximo e mínimo de $4,5 \%$ e $2,3 \%$, respectivamente, no estudo da seleção de progênies de maracujazeiro-amarelo para a região Norte. Para Negreiros et al. (2008), os valores de ATT variaram de 4,8 a $3,2 \%$, também no estudo de diferentes genótipos.

No presente trabalho, a relação SST/ATT variou de 4,3 a 2,6 (Tabela 2). Essa relação pode ser influenciada por fatores ambientais, como intensidade de luz solar e temperatura, como por tipo e doses de fertilizantes (NASCIMENTO et al., 2003).A análise do balanço entre açúcares e ácidos no fruto (relação SST/ATT) tem utilidade na avaliação do sabor e na determinação de estádios de maturação, uma vez que seu valor tende a aumentar à medida que o fruto vai atingindo o ponto de colheita (CHITARRA; CHITARRA, 2005). 
TABELA 1 - Produção, número de frutos por planta e massa média dos frutos no $1^{\circ} \mathrm{e} 2^{\circ}$ ciclos de 36 híbridos de maracujazeiro-amarelo no norte do Paraná. Londrina-PR, 2007 e 2008.

\begin{tabular}{|c|c|c|c|c|c|c|c|c|c|}
\hline \multirow{2}{*}{ Híbridos } & \multicolumn{3}{|c|}{ Produção } & \multicolumn{3}{|c|}{$N^{0}$ de frutos } & \multicolumn{3}{|c|}{ Massa média dos frutos } \\
\hline & $1^{0}$ ciclo & $2^{0}$ ciclo & Acum. & $\mathbf{1}^{0}$ ciclo & $2^{\circ}$ ciclo & Acum. & $1^{\circ}$ ciclo & $2^{0}$ ciclo & Média \\
\hline & \multicolumn{3}{|c|}{--------kg planta-1'--------- } & & & & & $----g--$ & ------ \\
\hline 29 & $25,1 \mathrm{ab}^{*}$ & $60,0 \mathrm{a}$ & $85,0 \mathrm{a}$ & $172,7 \mathrm{ab}$ & $413,3 \mathrm{a}$ & $586,0 \mathrm{a}$ & $144,2 \mathrm{bc}$ & $145,7 \mathrm{ab}$ & $145,0 \mathrm{ab}$ \\
\hline 30 & $21,9 \mathrm{ab}$ & $58,4 \mathrm{a}$ & $80,2 \mathrm{a}$ & $125,7 \mathrm{ab}$ & $377,3 \mathrm{a}$ & $503,0 \mathrm{a}$ & $173,8 \mathrm{abc}$ & $153,7 \mathrm{ab}$ & $163,7 \mathrm{ab}$ \\
\hline 31 & $19,9 \mathrm{~b}$ & $75,0 \mathrm{a}$ & 94,9 a & $102,3 \mathrm{~b}$ & $413,7 \mathrm{a}$ & $516,0 \mathrm{a}$ & $197,1 \mathrm{ab}$ & $181,2 \mathrm{ab}$ & $189,2 \mathrm{ab}$ \\
\hline 32 & $26,4 \mathrm{ab}$ & $75,6 \mathrm{a}$ & $102,1 \mathrm{a}$ & $134,0 \mathrm{ab}$ & $421,0 \mathrm{a}$ & $555,0 \mathrm{a}$ & $196,8 \mathrm{ab}$ & $182,0 \mathrm{ab}$ & $189,4 \mathrm{ab}$ \\
\hline 33 & $24,6 \mathrm{ab}$ & $61,8 \mathrm{a}$ & 86,4 a & $144,7 \mathrm{ab}$ & $372,5 \mathrm{a}$ & $517,0 \mathrm{a}$ & $170,5 \mathrm{abc}$ & $166,8 \mathrm{ab}$ & $168,6 \mathrm{ab}$ \\
\hline 34 & $26,4 \mathrm{ab}$ & $67,5 \mathrm{a}$ & $93,9 \mathrm{a}$ & $153,7 \mathrm{ab}$ & $432,0 \mathrm{a}$ & $585,7 \mathrm{a}$ & $181,9 \mathrm{abc}$ & $157,9 \mathrm{ab}$ & $169,9 a b$ \\
\hline 35 & $23,7 \mathrm{ab}$ & $62,8 \mathrm{a}$ & 78,6 a & $141,0 \mathrm{ab}$ & $420,0 \mathrm{a}$ & $514,0 \mathrm{a}$ & $168,4 \mathrm{abc}$ & $149,0 \mathrm{ab}$ & $130,6 \mathrm{~b}$ \\
\hline 36 & $23,0 \mathrm{ab}$ & $69,1 \mathrm{a}$ & $92,1 \mathrm{a}$ & $121,0 \mathrm{ab}$ & $475,0 \mathrm{a}$ & $596,0 \mathrm{a}$ & $190,2 \mathrm{abc}$ & $145,5 \mathrm{ab}$ & $167,8 a b$ \\
\hline 37 & $28,3 \mathrm{ab}$ & $80,4 \mathrm{a}$ & $108,7 \mathrm{a}$ & $159,3 \mathrm{ab}$ & $a$ & $634,7 \mathrm{a}$ & $178,7 \mathrm{abc}$ & $168,2 \mathrm{ab}$ & $173,5 \mathrm{ab}$ \\
\hline 38 & $27,3 \mathrm{ab}$ & $73,5 \mathrm{a}$ & $100,9 \mathrm{a}$ & $154,7 \mathrm{ab}$ & $417,0 \mathrm{a}$ & $571,7 \mathrm{a}$ & $173,4 \mathrm{abc}$ & $180,1 \mathrm{ab}$ & $176,7 a b$ \\
\hline 39 & $24,4 \mathrm{ab}$ & 56,4 a & 80,8 a & $138,3 \mathrm{ab}$ & $359,0 \mathrm{a}$ & $497,3 \mathrm{a}$ & $181,5 \mathrm{abc}$ & $159,3 \mathrm{ab}$ & $170,4 \mathrm{ab}$ \\
\hline 40 & $39,5 \mathrm{a}$ & $70,6 \mathrm{a}$ & $110,1 \mathrm{a}$ & $209,7 \mathrm{ab}$ & $404,0 \mathrm{a}$ & $543,7 \mathrm{a}$ & $189,8 \mathrm{abc}$ & $173,8 \mathrm{ab}$ & $179,6 \mathrm{ab}$ \\
\hline 41 & $23,6 \mathrm{ab}$ & $72,9 \mathrm{a}$ & $96,5 \mathrm{a}$ & $127,0 \mathrm{ab}$ & $431,7 \mathrm{a}$ & $558,7 \mathrm{a}$ & $186,3 \mathrm{abc}$ & $171,4 \mathrm{ab}$ & $178,9 \mathrm{ab}$ \\
\hline 42 & $23,8 \mathrm{ab}$ & $79,3 \mathrm{a}$ & $103,1 \mathrm{a}$ & $127,3 \mathrm{ab}$ & $471,0 \mathrm{a}$ & $598,3 \mathrm{a}$ & $186,8 \mathrm{abc}$ & $165,1 \mathrm{ab}$ & $175,9 a b$ \\
\hline 43 & $37,6 \mathrm{ab}$ & $63,8 \mathrm{a}$ & $101,4 \mathrm{a}$ & $228,0 \mathrm{a}$ & $452,0 \mathrm{a}$ & $680,0 \mathrm{a}$ & $166,5 \mathrm{abc}$ & $139,1 \mathrm{~b}$ & $152,8 \mathrm{ab}$ \\
\hline 44 & $26,8 \mathrm{ab}$ & $61,3 \mathrm{a}$ & $88,1 \mathrm{a}$ & $158,7 \mathrm{ab}$ & $368,7 \mathrm{a}$ & $527,3 \mathrm{a}$ & $169,5 \mathrm{abc}$ & $163,5 \mathrm{ab}$ & $166,5 \mathrm{ab}$ \\
\hline 45 & $34,4 \mathrm{ab}$ & $71,8 \mathrm{a}$ & $106,1 \mathrm{a}$ & $212,7 \mathrm{ab}$ & $446,7 \mathrm{a}$ & $659,0 \mathrm{a}$ & $161,6 \mathrm{abc}$ & $160,0 \mathrm{ab}$ & $160,8 \mathrm{ab}$ \\
\hline 46 & $27,5 \mathrm{ab}$ & $71,8 \mathrm{a}$ & $99,3 \mathrm{a}$ & $147,3 \mathrm{ab}$ & $453,3 \mathrm{a}$ & $600,7 \mathrm{a}$ & $191,7 \mathrm{abc}$ & $163,3 \mathrm{ab}$ & $177,5 \mathrm{ab}$ \\
\hline 47 & $30,3 \mathrm{ab}$ & $62,7 \mathrm{a}$ & $93,0 \mathrm{a}$ & $192,7 \mathrm{ab}$ & $415,7 \mathrm{a}$ & $608,3 \mathrm{a}$ & $156,6 \mathrm{abc}$ & $150,1 \mathrm{ab}$ & $153,4 a b$ \\
\hline 48 & $25,0 \mathrm{ab}$ & $80,5 \mathrm{a}$ & $105,4 \mathrm{a}$ & $130,3 \mathrm{ab}$ & $429,0 \mathrm{a}$ & 559,3 a & $191,2 \mathrm{abc}$ & $187,0 \mathrm{ab}$ & $189,1 \mathrm{ab}$ \\
\hline 49 & $19,7 \mathrm{~b}$ & $54,0 \mathrm{a}$ & $73,8 \mathrm{a}$ & $111,0 \mathrm{~b}$ & $365,0 \mathrm{a}$ & $476,0 \mathrm{a}$ & $179,0 \mathrm{abc}$ & $149,2 \mathrm{ab}$ & $164,1 \mathrm{ab}$ \\
\hline 50 & $31,4 \mathrm{ab}$ & $96,0 \mathrm{a}$ & $127,4 \mathrm{a}$ & $173,7 \mathrm{ab}$ & $554,3 \mathrm{a}$ & $728,0 \mathrm{a}$ & $181,3 \mathrm{abc}$ & $172,8 \mathrm{ab}$ & $177,0 \mathrm{ab}$ \\
\hline 51 & $20,8 \mathrm{~b}$ & $66,8 \mathrm{a}$ & $82,2 \mathrm{a}$ & 110,5 b & $394,3 \mathrm{a}$ & $476,7 \mathrm{a}$ & $188,6 \mathrm{abc}$ & $169,1 \mathrm{ab}$ & $177,6 \mathrm{ab}$ \\
\hline 52 & $25,5 \mathrm{ab}$ & $77,2 \mathrm{a}$ & 87,6 a & $152,7 \mathrm{ab}$ & $442,0 \mathrm{a}$ & $474,0 \mathrm{a}$ & $171,8 \mathrm{abc}$ & $179,4 \mathrm{ab}$ & $164,3 \mathrm{ab}$ \\
\hline 53 & $22,3 \mathrm{ab}$ & $68,8 \mathrm{a}$ & $91,0 \mathrm{a}$ & $119,0 \mathrm{ab}$ & $383,7 \mathrm{a}$ & $502,7 \mathrm{a}$ & $186,1 \mathrm{abc}$ & $177,9 \mathrm{ab}$ & $182,0 \mathrm{ab}$ \\
\hline 54 & $23,9 \mathrm{ab}$ & $70,2 \mathrm{a}$ & $94,1 \mathrm{a}$ & $155,3 \mathrm{ab}$ & $490,3 \mathrm{a}$ & $645,7 \mathrm{a}$ & $153,7 \mathrm{abc}$ & $143,9 \mathrm{ab}$ & $148,8 \mathrm{ab}$ \\
\hline 55 & $25,2 \mathrm{ab}$ & $82,3 \mathrm{a}$ & $107,5 \mathrm{a}$ & $142,0 \mathrm{ab}$ & $517,3 \mathrm{a}$ & 659,3 a & $184,1 \mathrm{abc}$ & $161,3 \mathrm{ab}$ & $172,7 \mathrm{ab}$ \\
\hline 56 & $29,2 \mathrm{ab}$ & $72,2 \mathrm{a}$ & $101,4 \mathrm{a}$ & $169,7 \mathrm{ab}$ & $456,7 \mathrm{a}$ & $626,3 \mathrm{a}$ & $171,7 \mathrm{abc}$ & $158,3 \mathrm{ab}$ & $165,0 \mathrm{ab}$ \\
\hline 57 & $25,0 \mathrm{ab}$ & $53,1 \mathrm{a}$ & $78,0 \mathrm{a}$ & $129,3 \mathrm{ab}$ & $377,3 \mathrm{a}$ & $506,7 \mathrm{a}$ & $196,2 \mathrm{ab}$ & $133,5 \mathrm{~b}$ & $164,8 a b$ \\
\hline 58 & $24,3 \mathrm{ab}$ & $63,6 \mathrm{a}$ & $88,0 \mathrm{a}$ & $133,5 \mathrm{ab}$ & $389,7 \mathrm{a}$ & $383,3 \mathrm{a}$ & $182,3 \mathrm{abc}$ & $163,1 \mathrm{ab}$ & $161,4 \mathrm{ab}$ \\
\hline 59 & $21,2 \mathrm{~b}$ & $53,6 \mathrm{a}$ & $74,7 \mathrm{a}$ & $143,3 \mathrm{ab}$ & $379,0 \mathrm{a}$ & $522,3 \mathrm{a}$ & $149,4 \mathrm{abc}$ & $142,5 \mathrm{ab}$ & $146,0 \mathrm{ab}$ \\
\hline 60 & $27,1 \mathrm{ab}$ & $71,0 \mathrm{a}$ & $98,1 \mathrm{a}$ & $153,7 \mathrm{ab}$ & $430,0 \mathrm{a}$ & $583,7 \mathrm{a}$ & $177,7 \mathrm{abc}$ & $165,6 \mathrm{ab}$ & $171,7 \mathrm{ab}$ \\
\hline 61 & $27,6 \mathrm{ab}$ & $73,8 \mathrm{a}$ & $101,5 \mathrm{a}$ & $198,0 \mathrm{ab}$ & $505,3 \mathrm{a}$ & $703,3 \mathrm{a}$ & $138,3 \mathrm{c}$ & $144,9 \mathrm{ab}$ & $141,6 \mathrm{~b}$ \\
\hline 62 & $28,7 \mathrm{ab}$ & $54,6 \mathrm{a}$ & 83,3 a & $163,7 \mathrm{ab}$ & $373,3 \mathrm{a}$ & $537,0 \mathrm{a}$ & $174,8 \mathrm{abc}$ & $147,8 \mathrm{ab}$ & $161,3 \mathrm{ab}$ \\
\hline 63 & $23,9 \mathrm{ab}$ & $72,4 \mathrm{a}$ & 96,3 a & $116,3 \mathrm{ab}$ & $368,0 \mathrm{a}$ & $484,3 \mathrm{a}$ & $205,3 \mathrm{a}$ & 199,3 a & 202,3 a \\
\hline 64 & $29,3 \mathrm{ab}$ & $75,7 \mathrm{a}$ & $105,0 \mathrm{a}$ & $194,0 \mathrm{ab}$ & $536,7 \mathrm{a}$ & $730,7 \mathrm{a}$ & $154,1 \mathrm{abc}$ & $143,3 \mathrm{ab}$ & $148,7 \mathrm{ab}$ \\
\hline CV $(\%)$ & 21,13 & 21,05 & 19,32 & 22,90 & 19,99 & 19,77 & 9,72 & 10,86 & 10,75 \\
\hline
\end{tabular}

*Médias seguidas da mesma letra nas colunas não diferem entre si, pelo teste de Tukey, a 5\% de significância. Acum.: acumulado dos dois ciclos. 
TABELA 2 - Comprimento, diâmetro, relação entre comprimento e diâmetro (C/D), espessura da casca, rendimento de polpa, teor de sólidos solúveis totais (SST), acidez total titulável (ATT) e relação entre o teor de sólidos solúveis totais e acidez total titulável (SST/ATT) dos frutos de 36 híbridos de maracujazeiro-amarelo, no norte do Paraná. Médias dos dois ciclos. LondrinaPR, 2007 e 2008.

\begin{tabular}{|c|c|c|c|c|c|c|c|c|}
\hline Híbridos & Comp. & Diâm. & C/D & Espessura & Rend.** & SST & ATT & SST/ATT \\
\hline & & & & & ---\%--- & --'Brix-- & --\%-- & \\
\hline 29 & $79,7 a^{*}$ & $72,6 \mathrm{a}$ & $1,10 \mathrm{abc}$ & 5,0 bcde & $26,6 \mathrm{ab}$ & $13,8 \mathrm{a}$ & $3.6 \mathrm{ab}$ & $3,9 \mathrm{abc}$ \\
\hline 30 & $83,8 \mathrm{a}$ & $75,3 \mathrm{a}$ & $1,11 \mathrm{abc}$ & 5,0 bcde & $27,5 \mathrm{ab}$ & $14,2 \mathrm{a}$ & $3,8 \mathrm{ab}$ & $3,8 \mathrm{abc}$ \\
\hline 31 & $92,8 \mathrm{a}$ & $79,3 \mathrm{a}$ & $1,17 \mathrm{abc}$ & 5,5 abcde & $29,7 \mathrm{ab}$ & $14,9 \mathrm{a}$ & $3,9 \mathrm{ab}$ & $3,8 \mathrm{abc}$ \\
\hline 32 & $84,7 \mathrm{a}$ & $82,8 \mathrm{a}$ & $1,02 \mathrm{c}$ & $7,6 \mathrm{a}$ & $22,0 \mathrm{ab}$ & $13,0 \mathrm{a}$ & $3,2 \mathrm{~b}$ & $4,1 \mathrm{ab}$ \\
\hline 33 & $91,7 \mathrm{a}$ & $81,8 \mathrm{a}$ & $1,12 \mathrm{abc}$ & 4,7 bcde & $28,3 \mathrm{ab}$ & $13,9 \mathrm{a}$ & $4,2 \mathrm{ab}$ & $3,3 a b c$ \\
\hline 34 & $91,0 \mathrm{a}$ & $79,3 \mathrm{a}$ & $1,15 \mathrm{abc}$ & 4,8 bcde & $31,9 \mathrm{ab}$ & $14,1 \mathrm{a}$ & $4,6 \mathrm{ab}$ & $3,1 \mathrm{abc}$ \\
\hline 35 & $78,9 \mathrm{a}$ & $64,5 \mathrm{a}$ & $1,20 \mathrm{abc}$ & $3,6 \mathrm{de}$ & $25,5 \mathrm{ab}$ & $10,8 \mathrm{a}$ & $3,1 \mathrm{~b}$ & $2,9 a b c$ \\
\hline 36 & $95,8 \mathrm{a}$ & $76,5 \mathrm{a}$ & $1,25 \mathrm{a}$ & 4,3 bcde & $29,2 \mathrm{ab}$ & $12,9 \mathrm{a}$ & $4,2 \mathrm{ab}$ & $3,1 \mathrm{abc}$ \\
\hline 37 & $92,8 \mathrm{a}$ & $80,8 \mathrm{a}$ & $1,15 \mathrm{abc}$ & 4,8 bcde & $27,9 \mathrm{ab}$ & $14,5 \mathrm{a}$ & $4,2 \mathrm{ab}$ & $3,5 \mathrm{abc}$ \\
\hline 38 & $91,9 \mathrm{a}$ & $78,5 \mathrm{a}$ & $1,17 \mathrm{abc}$ & 5,4 abcde & $28,1 \mathrm{ab}$ & $14,6 \mathrm{a}$ & $3,5 \mathrm{ab}$ & $4,3 \mathrm{a}$ \\
\hline 39 & $93,4 \mathrm{a}$ & $82,8 \mathrm{a}$ & $1,13 \mathrm{abc}$ & 4,9 bcde & $30,4 \mathrm{ab}$ & $14,0 \mathrm{a}$ & $4,4 \mathrm{ab}$ & $3,1 \mathrm{abc}$ \\
\hline 40 & $76,4 \mathrm{a}$ & $71,4 \mathrm{a}$ & $1,08 \mathrm{abc}$ & 4,2 bcde & $22,1 \mathrm{ab}$ & $12,0 \mathrm{a}$ & $3,4 \mathrm{~b}$ & $2,9 \mathrm{abc}$ \\
\hline 41 & $90,6 \mathrm{a}$ & $81.1 \mathrm{a}$ & $1.12 \mathrm{abc}$ & 5,7 abcde & $27.5 \mathrm{ab}$ & $14,2 \mathrm{a}$ & $3,5 \mathrm{~b}$ & $4,2 \mathrm{ab}$ \\
\hline 42 & $92,6 \mathrm{a}$ & $82,3 \mathrm{a}$ & $1,12 \mathrm{abc}$ & $6,7 \mathrm{ab}$ & $19,6 \mathrm{~b}$ & $13,0 \mathrm{a}$ & $3,2 \mathrm{~b}$ & $4,1 \mathrm{ab}$ \\
\hline 43 & $91,3 \mathrm{a}$ & $75,3 \mathrm{a}$ & $1,21 \mathrm{ab}$ & $4,7 \mathrm{bcde}$ & $35,5 \mathrm{a}$ & $12,9 \mathrm{a}$ & $5,1 \mathrm{a}$ & $2,6 \mathrm{c}$ \\
\hline 44 & $87,9 \mathrm{a}$ & $78,0 \mathrm{a}$ & $1,13 \mathrm{abc}$ & 5,5 abcde & $23,2 \mathrm{ab}$ & $13,5 \mathrm{a}$ & $3,9 \mathrm{ab}$ & $3,5 \mathrm{abc}$ \\
\hline 45 & $85,9 \mathrm{a}$ & $80,3 \mathrm{a}$ & $1,07 \mathrm{bc}$ & 5,4 abcde & $25,8 \mathrm{ab}$ & $13,9 \mathrm{a}$ & $3,9 \mathrm{ab}$ & $3,6 a b c$ \\
\hline 46 & $91,1 \mathrm{a}$ & $76,8 \mathrm{a}$ & $1,19 \mathrm{abc}$ & 5,0 bcde & $31,2 \mathrm{ab}$ & $15,2 \mathrm{a}$ & $3,9 \mathrm{ab}$ & $4,0 \mathrm{ab}$ \\
\hline 47 & $87,3 \mathrm{a}$ & $78,1 \mathrm{a}$ & $1,12 \mathrm{abc}$ & 5,1 abcde & $28,1 \mathrm{ab}$ & $12,2 \mathrm{a}$ & $3,9 \mathrm{ab}$ & $3,1 \mathrm{abc}$ \\
\hline 48 & $91,6 \mathrm{a}$ & $80,9 \mathrm{a}$ & $1,13 \mathrm{abc}$ & 5,2 abcde & $26,6 \mathrm{ab}$ & $15,2 \mathrm{a}$ & $4,3 \mathrm{ab}$ & $3,6 a b c$ \\
\hline 49 & $92,5 \mathrm{a}$ & $78,3 \mathrm{a}$ & $1,18 \mathrm{abc}$ & 5,2 abcde & $32,4 \mathrm{ab}$ & $12,1 \mathrm{a}$ & $4,0 \mathrm{ab}$ & $3,1 \mathrm{abc}$ \\
\hline 50 & $86,1 \mathrm{a}$ & $81,3 \mathrm{a}$ & $1,06 \mathrm{bc}$ & 4,9 bcde & $27,1 \mathrm{ab}$ & $13,1 \mathrm{a}$ & $3,8 \mathrm{ab}$ & $3,5 \mathrm{abc}$ \\
\hline 51 & $91,1 \mathrm{a}$ & $82,4 \mathrm{a}$ & $1,10 \mathrm{abc}$ & 5,6 abcde & $23,6 \mathrm{ab}$ & $12,7 \mathrm{a}$ & $3,4 \mathrm{~b}$ & $3,8 \mathrm{abc}$ \\
\hline 52 & $85,3 \mathrm{a}$ & $78,7 \mathrm{a}$ & $1,08 \mathrm{abc}$ & $6,2 \mathrm{abc}$ & $29,2 \mathrm{ab}$ & $11,9 \mathrm{a}$ & $4,0 \mathrm{ab}$ & $3,0 \mathrm{abc}$ \\
\hline 53 & $84,4 \mathrm{a}$ & $77,0 \mathrm{a}$ & $1,10 \mathrm{abc}$ & 5,6 abcde & $28,7 \mathrm{ab}$ & $13,8 \mathrm{a}$ & $3,4 \mathrm{~b}$ & $4,1 \mathrm{ab}$ \\
\hline 54 & $94,1 \mathrm{a}$ & $82,2 \mathrm{a}$ & $1,15 \mathrm{abc}$ & 3,3 e & $31,6 \mathrm{ab}$ & $14,3 \mathrm{a}$ & $4,3 \mathrm{ab}$ & $3,3 \mathrm{abc}$ \\
\hline 55 & $93,0 \mathrm{a}$ & $77,0 \mathrm{a}$ & $1,21 \mathrm{ab}$ & 5,6 abcde & $28,3 \mathrm{ab}$ & $14,7 \mathrm{a}$ & $4,3 \mathrm{ab}$ & $3,4 \mathrm{abc}$ \\
\hline 56 & $78,9 \mathrm{a}$ & $75,8 \mathrm{a}$ & $1,04 \mathrm{bc}$ & 5,2 abcde & $33,3 \mathrm{ab}$ & $13,7 \mathrm{a}$ & $4.3 \mathrm{ab}$ & $3,2 \mathrm{abc}$ \\
\hline 57 & $90,4 \mathrm{a}$ & $75,6 \mathrm{a}$ & $1,20 \mathrm{ab}$ & 5,3 abcde & $24,4 \mathrm{ab}$ & $12,7 \mathrm{a}$ & $4,5 \mathrm{ab}$ & $3,0 \mathrm{abc}$ \\
\hline 58 & $74,7 \mathrm{a}$ & $64,5 \mathrm{a}$ & $1,17 \mathrm{abc}$ & 3,9 cde & $26,2 \mathrm{ab}$ & $11,0 \mathrm{a}$ & $3,3 \mathrm{~b}$ & $2,8 \mathrm{bc}$ \\
\hline 59 & $89,5 \mathrm{a}$ & $78,1 \mathrm{a}$ & $1,15 \mathrm{abc}$ & 4,5 bcde & $24,9 \mathrm{ab}$ & $12,5 \mathrm{a}$ & $3,7 \mathrm{ab}$ & $3,5 \mathrm{abc}$ \\
\hline 60 & $93,9 \mathrm{a}$ & $81.0 \mathrm{a}$ & $1.15 \mathrm{abc}$ & 4,4 bcde & $34.9 \mathrm{a}$ & $14.7 \mathrm{a}$ & $4,4 \mathrm{ab}$ & $3,4 \mathrm{abc}$ \\
\hline 61 & $87,4 \mathrm{a}$ & $76,4 \mathrm{a}$ & $1,14 \mathrm{abc}$ & $4,1 \mathrm{cde}$ & $34,1 \mathrm{ab}$ & $13,1 \mathrm{a}$ & $4,2 \mathrm{ab}$ & $3,1 \mathrm{abc}$ \\
\hline 62 & $\begin{array}{l}97,4 \mathrm{a} \\
90,8 \mathrm{a}\end{array}$ & $75,7 \mathrm{a}$ & $1,20 \mathrm{ab}$ & 4,7 bcde & $27,8 \mathrm{ab}$ & $12,6 \mathrm{a}$ & $3,7 \mathrm{ab}$ & 3,4 abc \\
\hline 63 & $93,4 \mathrm{a}$ & $83,8 \mathrm{a}$ & $1,11 \mathrm{abc}$ & $6,0 \mathrm{abcd}$ & $25,6 \mathrm{ab}$ & $14,4 \mathrm{a}$ & $3,6 \mathrm{ab}$ & $4,0 \mathrm{abc}$ \\
\hline 64 & $86,4 \mathrm{a}$ & $80,0 \mathrm{a}$ & $1,08 \mathrm{abc}$ & 5,2 abcde & $28,9 \mathrm{ab}$ & $12,5 \mathrm{a}$ & $3,9 \mathrm{ab}$ & $3,2 \mathrm{abc}$ \\
\hline $\mathrm{CV}(\%)$ & 10.05 & 9.59 & 4.72 & 15.61 & 9,32 & 1036 & 1241 & 1282 \\
\hline
\end{tabular}

*Médias seguidas da mesma letra nas colunas não diferem entre si, pelo teste de Tukey, a 5\% de significância.

**Dados transformados por transformação angular.

\section{CONCLUSÕES}

1-Os híbridos '40' e '43'apresentam, respectivamente, maior produção e maior número de frutos no primeiro ciclo de produção. O híbrido ' 63 ' apresenta maior massa dos frutos. Entretanto, apesar dos destaques, as médias desses híbridos não diferem estatisticamente de vários outros híbridos.

2-Todos os híbridos de maracujazeiroamarelo avaliados apresentam características desejáveis tanto para o mercado in natura como para a indústria.
3-Os frutos do híbrido ' 36 ' destacam-se no atendimento conjunto das características desejáveis de qualidade para o consumo in natura, enquanto os híbridos ' 43 ' e ' 60 ' apresentam características importantes para a indústria de sucos.

\section{REFERÊNCIAS}

ABREU, S. de P. M.; PEIXOTO, J. R.; JUNQUEIRA, N. T. V.; SOUSA, M. A. de F. Características físicoquímicas de cinco genótipos de maracujazeiro-azedo cultivados no Distrito Federal. Revista Brasileira de Fruticultura, Jaboticabal, v. 31, n. 2, p. 487 491, 2009. 
AGUIAR, D. R. D.; SANTOS, C. C. F. Importância econômica e mercado. In: BRUCKNER, C. H.; PIÇANHA, M. C. (Ed.). Maracujá: tecnologia de produção, pós-colheita, agroindústria, mercado. Porto Alegre: Cinco Continentes, 2001. p. 9-32.

ARAÚJO, C. M.; GAVA,A. J.; ROBBS, P. G.; NEVES, J. F.; MAIA, P. C. B. Características industriais do maracujá (Passiflora edulis f. flavicarpa) e maturação do fruto. Pesquisa Agropecuária Brasileira, Brasilia, v. 9, n. 9, p. 65-69, 1974.

AOAC - Association of Official Analytical Chemists. Official methods of analysis of the Association of Official Analytical Chemists. $15^{\text {th }}$ ed. Arlington, 1990, v.1. p.685-1.213.

BHERING, S. B. Mapa de solos do Estado do Paraná. Rio de Janeiro: Embrapa Solos, 2007. (Documentos).

BRASIL. Ministério da Agricultura e do Abastecimento. Instrução Normativa ${ }^{\circ} 12$, de 4 de setembro de 2003. Diário Oficial da União, Brasília, 2003. Seção1, p.72-76.

BRASIL. Ministério da Agricultura, Pecuária e Abastecimento. Registro Nacional de Cultivares - RNC. Disponível em: <http://extranet.agricultura. gov.br/php/snpc/cultivarweb/cultivares_registradas. php>. Acesso em: 20 jun 2012.

BRUCKNER, C. H.; MELETTI, L. M. M.; OTONI, W. C.; ZERBINI JÚNIOR, F. M. Maracujazeiro. In. BRUCKNER, C.H. (Ed.). Melhoramento de fruteiras tropicais. Viçosa: UFV, 2002. p.373-410.

CAVIGLIONE, J. H.; KIIHL, L. R. B.; CARAMORI, P. H.; OLIVEIRA, D. Cartas climáticas do Paraná. Londrina: IAPAR, 2000. CD-ROM

Chitarra, M. I. F.; CHitARRA, A. B. Póscolheita de frutas e hortaliças: fisiologia e manuseio. 2. ed. Lavras: UFLA, 2005. p. 785.

DURIGAN, J. F.; SIGRIST, J. M. M.; ALVES, R. E.; FILGUEIRAS, H. A. C.; VIEIRA, G. Qualidade e tecnologia pós-colheita do maracujá. In: LIMA, A. DE A.; CUNHA, M. A. Produção e qualidade na passicultura. Cruz das Almas: Embrapa Mandioca e Fruticultura, 2004. 396p.
GODOY, R. C. B. de; LEDO, C. A. da S.; SANTOS, A. P. dos; MATOS, E. L. S.; LIMA, A. de A.; WASZCZYNSKYJ, N. Diversidade genética entre acessos de maracujazeiro- amarelo avaliada pelas características físico-químicas dos frutos. Revista Ceres, Viçosa, MG, v. 54, n. 316, p. 541-547, 2007.

GONÇALVES, G. M.; VIANA, A. P.; BEZERRA NETO, F. V.; PEREIRA, M. G.; PEREIRA, T. N. S. Seleção e herdabilidade na predição de ganhos genéticos em maracujá-amarelo. Pesquisa Agropecuária Brasileira, Brasília, v. 42, n. 2, p. 193-198, 2007.

IBGE - Instituto Brasileiro de Geografia e Estatística. Produção agrícola municipal. Banco de Dados Agregados. Disponível em: <www.sidra.ibge.gov. br>. Acesso em: 03 nov. 2011.

JUNQUEIRA， N. T. V.; SANTOS, E. C.; JUNQUEIRA, K. P.; FALEIRO, F. G.; BELLON, G.; BRAGA, M. F. Características físico-químicas e produtividade de acessos de Passiflora nitida Kunth procedentes do Centro-Norte do Brasil. Revista Brasileira de Fruticultura, Jaboticabal, v. 32, n. 3, p. 791-797, 2010.

LIMA, A. de A; CUNHA, M. A. P. Maracujá: produção e qualidade na passicultura. Cruz das Almas: Embrapa Mandioca e Fruticultura, 2004. 396p.

MAIA, T. E. G.; PEIXOTO, J. R.; JUNQUEIRA, N. T. V.; SOUSA, M. A. F. Desempenho agronômico de genótipos de maracujazeiro-azedo cultivados no Distrito Federal. Revista Brasileira de Fruticultura, Jaboticabal, v. 31, n. 2, p. 500-506, 2009.

MELETTI, L. M. M.; SANTOS, R. R. dos; MINAMI, K. Melhoramento do maracujazeiro-amarelo: obtenção do cultivar 'composto IAC-27'. Scientia Agricola, Piracicaba, v. 57, n. 3, p. 491-498, 2000.

MELO, K. T.; MANICA, I.; JUNQUEIRA, N. T. V. Produtividade de seis cultivares de maracujazeiroazedo durante três anos, em Vargem Bonita-DF. Pesquisa Agropecuária Brasileira, Brasília, v. 36, n. 9, p. 1.117-1.125, 2001.

NASCIMENTO, T. B.; RAMOS, J. D.; MENEZES, J. B. Características físicas do maracujá-amarelo produzido em diferentes épocas. Pesquisa Agropecuária Brasileira, Brasília, v. 34, n. 12, p. 2.353-2.358, 1999. 
NASCIMENTO, W. M. O.; TOMÉ,A. T.; OLIVEIRA, M. S. P.; MÜllER, C. H.; CARVALHO, J. E. U. Seleção de progênies de maracujazeiro-amarelo (Passiflora edulis f. flavicarpa) quanto à qualidade de frutos. Revista Brasileira de Fruticultura, Jaboticabal, v. 25, n. 1, p. 186-188, 2003.

NEGREIROS, J. R. S.; ARAÚJO NETO, S. E.; ÁlVARES, V. S.; LIMA, V. A. L.; OLIVEIRA, T. K. Caracterização de frutos de progênies de meios-irmãos de maracujazeiro-amarelo em Rio Branco - Acre. Revista Brasileira de Fruticultura, Jaboticabal, v. 30, n. 2, p.431-437, 2008.

PIO, R.; RAMOS, J. D.; MENDONÇA, V.; GONTIJO, T. C.A.; RUFINI, J. C. M.; JUNQUEIRA, K. P. Caracterização físico-química dos frutos de sete seleções de maracujazeiro-amarelo para a região de Lavras - MG. Revista Ceres, Viçosa, MG, v.50, n. 291, p. 573-582, 2003.
POLL, H.; BENNO, A. Z. V.; KIST, B.; SANTOS, C.; CARVAlHO, C.; REETZ, E. R.; BELING, R. R. Anuário brasileiro da fruticultura 2011. Santa Cruz do Sul: Editora Gazeta Santa Cruz, 2011.p. 128.

SOUZA, A. C. G. de; SANDI, D. Industrialização. In: BRUCKNER, C.H.; PICANÇO, M.C. Maracujá: tecnologia de produção, pós-colheita, agroindústria, mercado. Porto Alegre: Cinco Continentes, 2001. $472 \mathrm{p}$.

VERAS, M. C. M; PINTO, A. C. de Q.; MENESES, J. B. de. Influência da época de produção e dos estádios de maturação nos maracujás doce e ácido nas condições de cerrado. Pesquisa Agropecuária Brasileira, Brasília, v. 35, n. 5, p. 959-966, 2000. 